\title{
Comparison of Various Methods for Isolation of Nocardia from Soil
}

\author{
Masoumeh Rasouli-Nasab, ${ }^{1}$ Mehdi Fatahi-Bafghi, ${ }^{2}$ Shadi Habibnia, ${ }^{1}$ Parvin Heidarieh, ${ }^{3}$ and Seyyed
}

\author{
Saeed Eshraghi ${ }^{1, *}$ \\ ${ }^{1}$ Department of Pathobiology, School of Public Health, Tehran University of Medical Sciences, Tehran, IR Iran \\ ${ }^{2}$ Department of Microbiology, Faculty of Medicine, Shahid Sadoughi University of Medical Sciences, Yazd, IR Iran \\ ${ }^{3}$ Department of Bacteriology and Virology, School of Medicine, Alborz University of medical Sciences, Karaj, IR Iran \\ "Corresponding author: Seyyed Saeed Eshraghi, Department of Pathobiology, School of Public Health, Tehran University of Medical Sciences, Qods St., Poursina St., Tehran, IR \\ Iran. Tel: +98-2188994823, E-mail: eshraghs@tums.ac.ir
}

Received 2016 March 19; Revised 2016 August 17; Accepted 2017 February 08.

\begin{abstract}
Background: The genus Nocardia is Gram-positive, aerobic filamentous bacilli and saprophytic micro-organisms that can be isolated from freshwater, salt water, dust and decaying vegetation especially the soil. This study aimed to investigate the several media for to determine a suitable culture media with the ability to better for the isolation of Nocardia from soil.

Methods: In this study, 400 soil samples were collected from different areas from Iran. The soil samples were then cultured on the four culture media such as Humic acid vitamin B agar, Paraffin agar, Sabouraud dextrose agar supplemented whit cycloheximide and carbon-free broth containing paraffin rods and incubated at $35^{\circ} \mathrm{C}$. All of culture media investigated every 3 days for a month. Colonies suspicious to Nocardia were stained with Gram-stain, acid-fast and partially acid-fast and evaluated for resistance to lysozyme.

Results: From 400 soil samples, the number of 62, 10, 28 and 19 strains of Nocardia were isolated by paraffin rods, Humic Acid Vitamin B agar, Paraffin agar and Sabouraud dextrose agar whit cycloheximide, respectively. Most Nocardia strains were isolated using paraffin bait technique.

Conclusions: Isolation of Nocardia spp. is enhanced by using the paraffin baiting technique that relies on the selective ability of this micro-organism to metabolize paraffin.
\end{abstract}

Keywords: Humic Acid Vitamin B Agar, Paraffin Bait Technique, Paraffin Agar, Sabouraud Dextrose Agar, Soil, Nocardia

\section{Introduction}

Nocardia spp. are related to the group of microorganisms known as the aerobic actinomycetes [1,2]. These bacteria are beaded branching filaments, Gram-positive and partially acid-fast bacilli [1-4]. Nocardia infections can cause pulmonary disease in both immune-compromised and healthy individuals [4]. Nocardia infections are initiated by inhalation of the organisms or through traumatic(such as primary cutaneous Nocardial infection) [5, 6]. Nocardia species are found as saprophytic components in natural habitats such as soil, dust, fresh and saltwater or the surfaces of plants, so the presence of these agents in laboratory or respiratory samples may represents contamination or acquisition of infection from their natural environment that diagnosed with clinical signs, radiological finding and especially culture of Nocardia [1]. So far, Nocardia spp. has been isolated of soil and by culture media such as paraffin bait method, humic acid vitamin B agar, paraffin agar, sucrose-gradient centrifugation, Lowenstein-Jenson, gelatin agar (GA), conventional media such as blood agar (BA) and urea agar [7-11]. Nocardia growth is slowly on nonselective culture media, and has the ability to grow in a wide temperature range. Isolation of Nocardia is difficult due to its slow growth which can be covered by the other fast-growing microorganisms. This study was performed to compare the several culture medium for choose more suitable medium for the isolation of the genus Nocardia from soil samples. Therefore, to achieve this aim was investigated the culture medium containing humic acid vitamin B agar, paraffin baiting method, paraffin agar and Sabouraud dextrose agar with cycloheximide.

\section{Methods}

A total of 400 soil samples were collected from February 2011 to September 2014 in different regions of Iran (Table 1) with approximately $4 \mathrm{~cm}$ deep. The samples were transferred to the laboratory immediately. In this study, for isolation of Nocardia from soil samples was used of 4 medium such as Humic acid vitamin B agar (HV agar), carbon free broth carbon-free broth (CFB) containing paraffin rods, paraffin agar (PA) and Sabouraud dextrose agar(SDA) whit cycloheximide. The composition of the HA agar is including 1.0 g Humic acid dissolved in $10 \mathrm{~mL}$ of $0.2 \mathrm{~N} \mathrm{NaOH}$, 
$0.5 \mathrm{~g} \mathrm{Na}_{2} \mathrm{HPO}_{4}, 0.5 \mathrm{~g}, 1.71 \mathrm{~g} \mathrm{KCI}, 0.05 \mathrm{~g} \mathrm{MgSO}_{4} .7 \mathrm{H}_{2} \mathrm{O}, 0.02$ $\mathrm{g} \mathrm{CaCO}_{3}, 0.01 \mathrm{~g} \mathrm{FeSO}_{4} .7 \mathrm{H}_{2} \mathrm{O}$, B-vitamins , $50.0 \mathrm{mg}$ cycloheximide, 18.0 g Agar, 1.0 lit distilled water (7).

Carbon-free broth medium (CFB) was used in paraffin baiting method, in which the sterile paraffin-coated glass placed into CFB $[8,11]$. Sabouraud dextrose agar medium according to the manufacturer's instructions was prepared. To preparation paraffin agar (PA) medium, Paraffin was added to Carbon-free agar (CFA) [11]. All of media were sterilized at $121^{\circ} \mathrm{C}$ for 15 minutes. The rate of $0.1 \mathrm{~mL}$ of suspensions each soil sample was placed and spread with a sterile loop simultaneously. About $1 \mathrm{~mL}$ of inoculate (soil sample) was added to a test tube containing 5 mLof sterile carbon-free broth and a paraffin-coated glass. Then these plates were incubated at $35^{\circ} \mathrm{C}$ and microbial on each medium was evaluated during a month. Nutrient agar (NA) medium and Sabouraud dextrose agar (SDA) were used to purify isolates. Colonies were grown in $35^{\circ} \mathrm{C}$ within 2 to 7 days. Identification of the genus Nocardia was based on Gram-positive, acid-fast and partially acid-fast staining, resistant to lysozyme and morphological tests such as size, shape and aerial hyphae [12-14].

\section{Results}

After inoculum of soil samples to each culture media (carbon-free broth containing paraffin rods, paraffin agar, humic acid vitamin B agar and Sabouraud dextrose agar whit cycloheximide separately and incubated at $35^{\circ} \mathrm{C}$, Nocardia growth was observed within 1 to 4 weeks (Figure 1). The 119 Nocardia colonies were observed on the culture media in 75 soil samples. In our study, both nutrient agar (NA) and SDA (without cycloheximide) were used to obtain pure cultures of Nocardia isolates (Figure 2). Nocardia colonies have a variable appearance. Colony morphology of 3 - 10 days pure cultures was smooth and moist, plaster, star-shaped, chalking wrinkled whit the colors white, orange and pink, creamy (Figure 2). Nocardia isolates were Gram-positive, non-acid-fast, partially acid-fast (Figure 3) and resistant to lysozyme (Figure 4). A total of 400 collected soil samples, 62 strains of Nocardia (15.5\%) were isolated by paraffin bait, 10 isolates $(2.5 \%)$ by Humic acid vitamin B agar, 28 isolates using paraffin agar (7\% ) and 19 isolates using the Sabouraud dextrose agar with cycloheximide (4.75\%) (See Table 1). Growth of Nocardia spp. was observed on each of four media in 10 soil samples. Most Nocardia isolates were obtained from soil samples collected from Mazandaran (40\%) (Figure 5).

\section{Discussion}

Nocardia is an aerobic, non-motile, partially acid-fast, rod-shaped and beaded branching filaments bacterium [15]. The genus Nocardia was isolated first by Edmond Nocard in 1888 from a case of bovine farcy. The genus of Nocardia is found commonly throughout the world in soil. Various studies have demonstrated that isolated from soil, plants, dust, decaying vegetation, and decaying fecal deposits from animals $[8,16]$. Nocardia species cause a wide spectrum of diseases including pulmonary nocardiosis; cutaneous and subcutaneous nocardiosis $[1,17,18]$. In a study by DeCroos et al. ocular exposure to soil or plant matter was a common historical point in cases of Nocardia keratitis (48\%) and scleritis (45\%), respectively [5]. Nocardia produce the secondary metabolism such as antibiotics like tubelactomicin A. Therefore, isolation and identification of Nocardia species from natural habitat is important $[1,19]$. The numbers of Nocardia species are increasing therefore accurate identification of Nocardia spp. is crucial to treatment of the Nocardial infections and also to identify new species of Nocardia [20,21]. It is noteworthy that diagnosis through biochemical and phenotypic methods are not enough. It would be better that a molecular technique had been used. Isolation of microorganism is first step for the identification. Various methods include pretreatment techniques in combination with enrichment techniques and selective antimicrobial agents have been developed to isolation the actinomycetes genera from natural habitats [22]. Nocardia can grow on routine bacterial, fungal, or mycobacterial culture media [23]. Few studies have been carried out in the isolation of Nocardia from soil using various methods. Isolation these bacteria from a mixture samples can sometimes be facilitated by the use of suitable combination (In order to create the selective media). For example, isolation of Nocardia spp. is enhanced by the use of paraffin, while many microorganisms cannot be use of paraffin as the sole carbon source $[8,11]$. In a study by Khan et al. in 1997 were isolated $41 \%$ of strains of the genus Nocardia using the paraffin bait of 102 soil samples [22, 24]. In a study conducted in 2012, HA agar allowed the growth of the largest numbers of actinomycetes colonies belonging to each genus of Streptomyces, Micromonospora, Microbispora, Streptosporangium, Nocardia, Dactylosporangium, Microtetraspora and Thermomonospora on the plate. HV agar was as the sole source of carbon and nitrogen was developed [7] According to studies by Hayakawa et al, HV agar may be recommended for greater growth of actinomycetes because of the activation of spore germination of actinomycetes is may be considered. In our study, the 10 colonies of Nocardia were recovered on HV agar media that less capable for isolation of these bacteria to com- 
Table 1. The Number of Soil Samples and Isolates of Nocardia from Iran Cities

\begin{tabular}{|c|c|c|c|c|c|c|}
\hline \multirow[t]{2}{*}{ Source of Soil Samples } & \multirow{2}{*}{$\begin{array}{l}\text { The Number of Soil } \\
\text { Samples Collected }\end{array}$} & \multirow{2}{*}{$\begin{array}{l}\text { The Number of Positive } \\
\text { Samples of Soil }\end{array}$} & \multicolumn{4}{|c|}{ The Number of Nocardia Isolates in Each Culture Medium } \\
\hline & & & Paraffin Bait Technique & HV Agar & PA & $\begin{array}{c}\text { SDA whit } \\
\text { Cycloheximide }\end{array}$ \\
\hline \multicolumn{7}{|l|}{ North provinces } \\
\hline Gilan & 28 & 9 & 8 & 6 & 3 & 6 \\
\hline Mazandaran & 40 & 16 & 13 & 3 & 5 & 1 \\
\hline Golestan & 27 & 6 & 5 & 1 & 0 & 6 \\
\hline \multicolumn{7}{|l|}{ Southern Province } \\
\hline Khuzestan & 13 & 0 & 0 & 0 & 0 & 0 \\
\hline Hormozgan & 10 & 0 & 0 & 0 & 0 & 0 \\
\hline Bushehr & 16 & 2 & 2 & 0 & 0 & 0 \\
\hline Fars & 15 & 2 & 1 & 0 & 0 & 2 \\
\hline Kerman & 8 & 1 & 1 & 0 & 0 & 1 \\
\hline \multicolumn{7}{|l|}{ Eastern Province } \\
\hline North Khorasan & 15 & 1 & 0 & 0 & 0 & 1 \\
\hline Razavi Khorasan & 34 & 3 & 0 & 0 & 0 & 3 \\
\hline South Khorasan & 13 & 0 & 0 & 0 & 0 & 0 \\
\hline \multicolumn{7}{|l|}{ Western Province } \\
\hline Ardabil & 7 & 0 & 0 & 0 & 0 & 0 \\
\hline East Azerbaijan & 8 & 2 & 2 & 0 & 0 & 0 \\
\hline Kurdistan & 7 & 0 & 0 & 0 & 0 & 0 \\
\hline Kermanshah & 13 & 2 & 2 & 0 & 0 & 0 \\
\hline Lorestan & 5 & 0 & 0 & 0 & 0 & 0 \\
\hline Hamadan & 12 & 3 & 3 & 0 & 0 & 0 \\
\hline Zanjan & 7 & 1 & 1 & 0 & 0 & 0 \\
\hline \multicolumn{7}{|l|}{ Central provinces } \\
\hline Isfahan & 8 & 3 & 3 & 0 & 2 & 1 \\
\hline Semnan & 15 & 3 & 2 & 0 & 0 & 2 \\
\hline Qom & 13 & 0 & 1 & 0 & 0 & 0 \\
\hline Yazd & 14 & 1 & 6 & 0 & 2 & 2 \\
\hline $\begin{array}{l}\text { Markazi Province } \\
\text { (Arak) }\end{array}$ & 16 & 5 & 5 & 0 & 3 & 1 \\
\hline Alborz & 20 & 6 & 0 & 0 & 0 & 0 \\
\hline Tehran & 36 & 9 & 7 & 0 & 4 & 3 \\
\hline
\end{tabular}

Abbreviations: HV, Humic acid-vitamin agar; PA, Paraffin agar; SDA, Sabouraud Dextrose Agar whit cycloheximide.

pared with the other two (SDA and PA). In the present study, bacterial growth on the HV agar medium was observed at least three weeks to form small orange/ yellow colonies (see Figure 3B). In a study, paraffin agar medium was introduced an inexpensive and selective medium for isolation of Nocardia from clinical compared with modified thayer martin medium and Paraffin baiting techniques [25]. In current study, PA medium due to its fragile, oily surface and high pollution and also is not recommended for separation these bacteria (see Figure 3C). Nocardia growth on this medium was observed after the third weeks. Despite greater clarity and earlier appearance of Nocardia colonies 


\section{Figure 1. Nocardia Colonies in the Primary Culture}
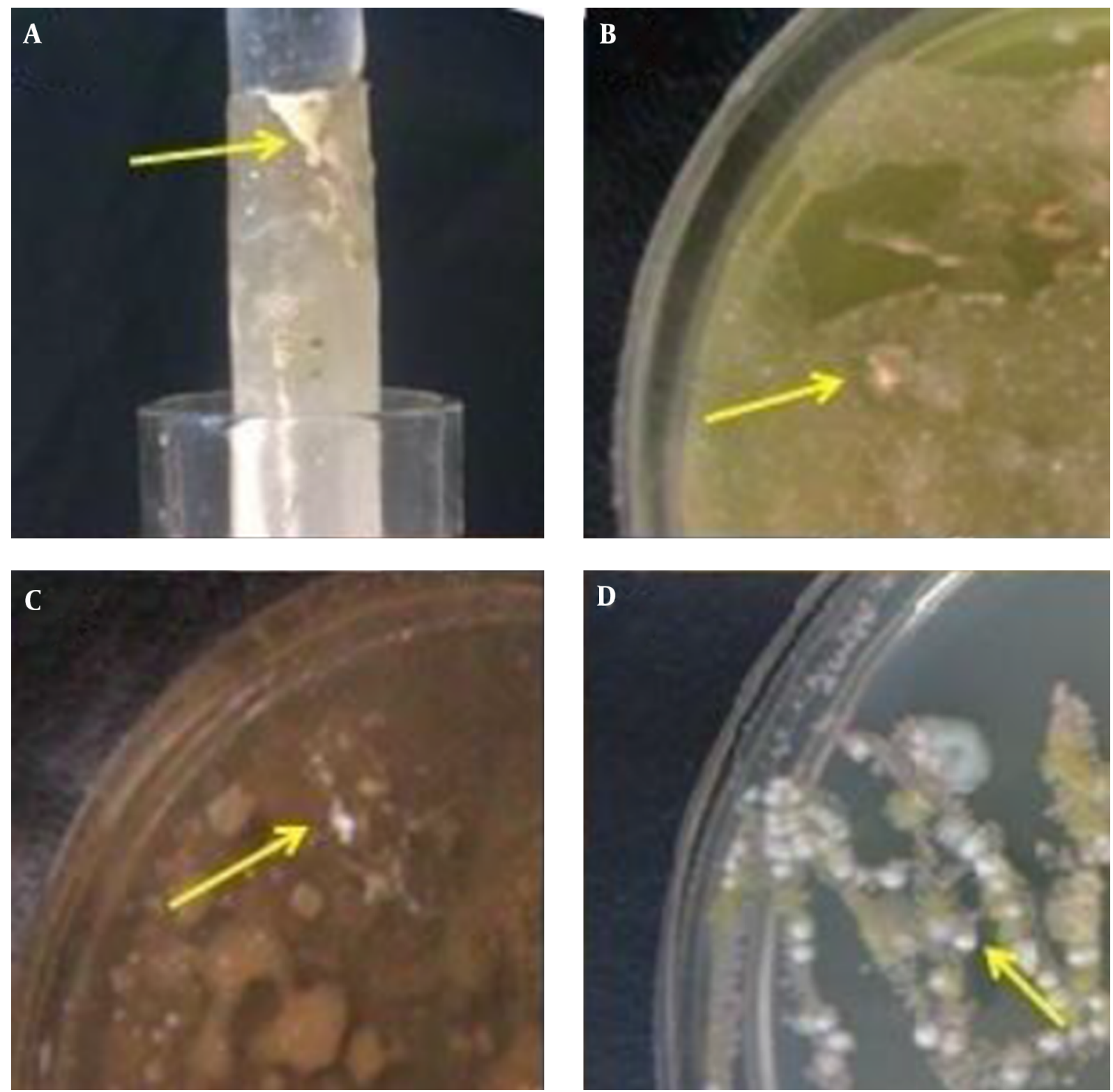

A; paraffin bait, B; HV agar, C; PA, D; SDA whit cycloheximide.

on SDA medium (See Figure 3D) than the PA medium, the low numbers of colonies were isolated on this media. In a study conducted by Aghamirian et al. 96 aerobic actinomycetes were isolated from 300 the soil samples of Qazvin province on brain-heart infusion agar and Sabouraud dextrose agar (SDA) [26]. In these cases, white and creamcolored growth was observed on the paraffin-coated rod above the surface of the carbon-free broth (See Figure 3A). The growth of the bacteria was observed on the paraffin coated rods and SDA earlier ( 2 to 4 weeks and in some cases in the first week). Plaster and white colonies of Nocardia were seen more on the paraffin rods in paraffin baiting media. In studies conducted by Shawar et al. Gugnani et al. and Bafghi et al. the paraffin bait technique can also be used for the recovery of Nocardia from clinical samples $[16,27,28]$. In our study the paraffin baiting technique is a more appropriate than other methods to isolation Nocardia from soil. Colonies of Nocardia in this tech- 

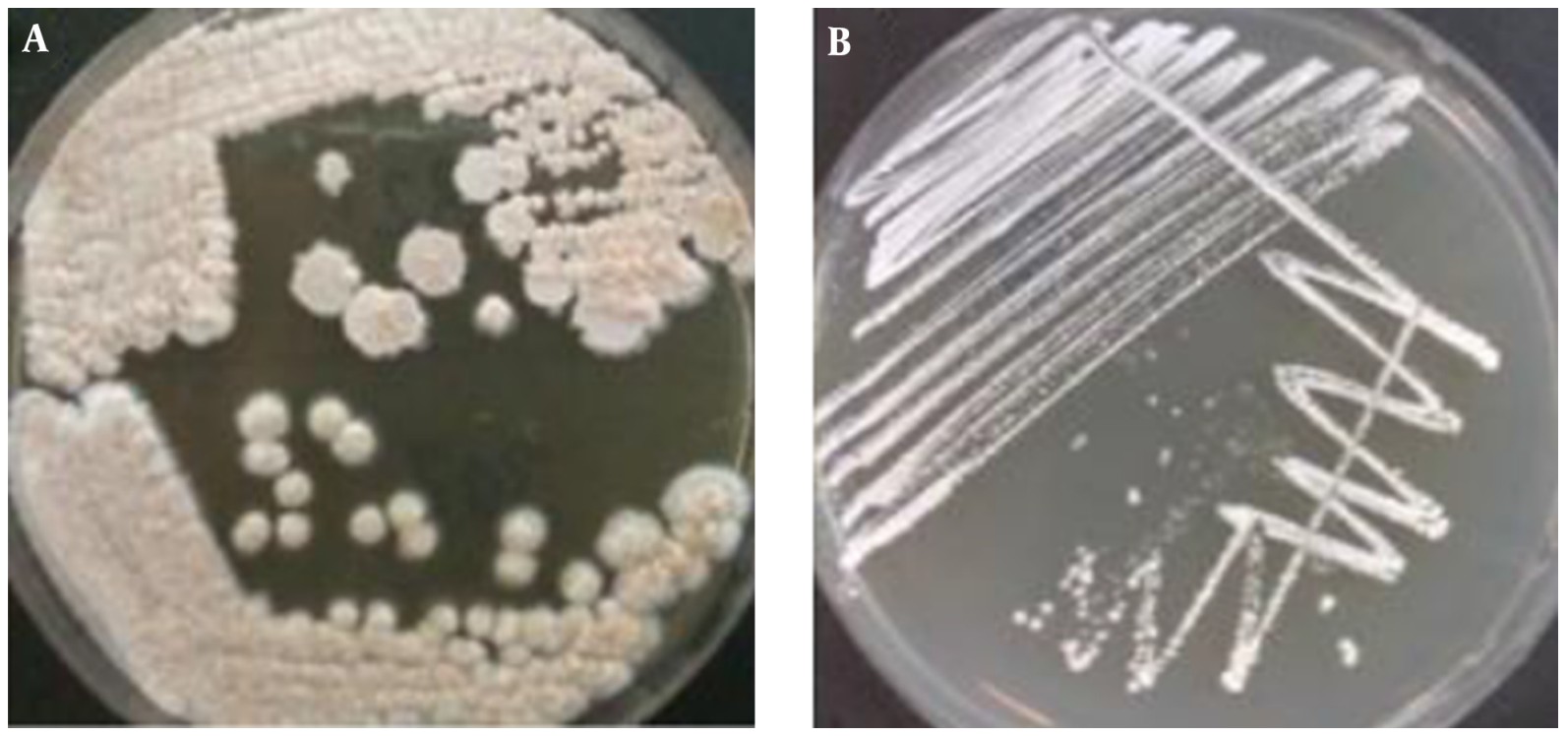

Figure 2. Pure Culture of Nocardia on: A, SDA; B, NA

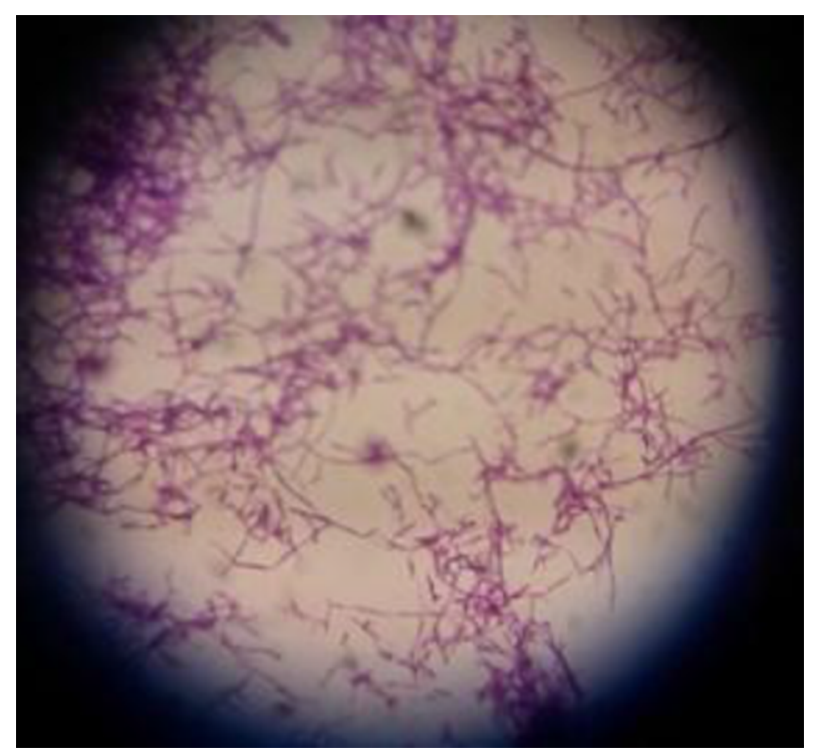

Figure 3. Partially Acid-Fast Beaded Branching Filaments

nique were detected better than other methods. The aim of this study was not to investigate the epidemiology, but we found that the most Nocardia species were isolated further on the northern edge (32.63\%) of the Iran country with moderate climate. It seemed that Nocardia growth in these areas has increased under the influence of factors such as organic matter, temperature, moisture, soil pH, soil nutrient cycling, vegetation and climatic conditions also. So, No-

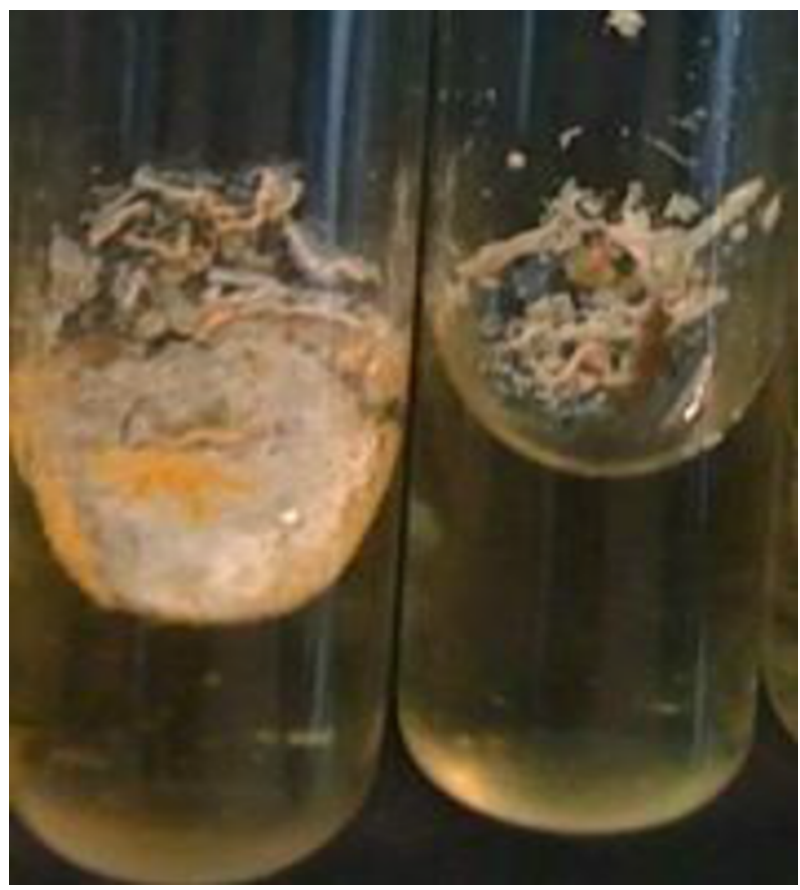

Figure 4. Growth of Nocardia in Lysozyme Broth (Resistant to Lysozyme)

cardia species is varied in different geographic locations. See the percent of isolation of Nocardia by each of the culture media in different geographic regions of Iran in Table 1 . It is seems, some selective medium are better for the 


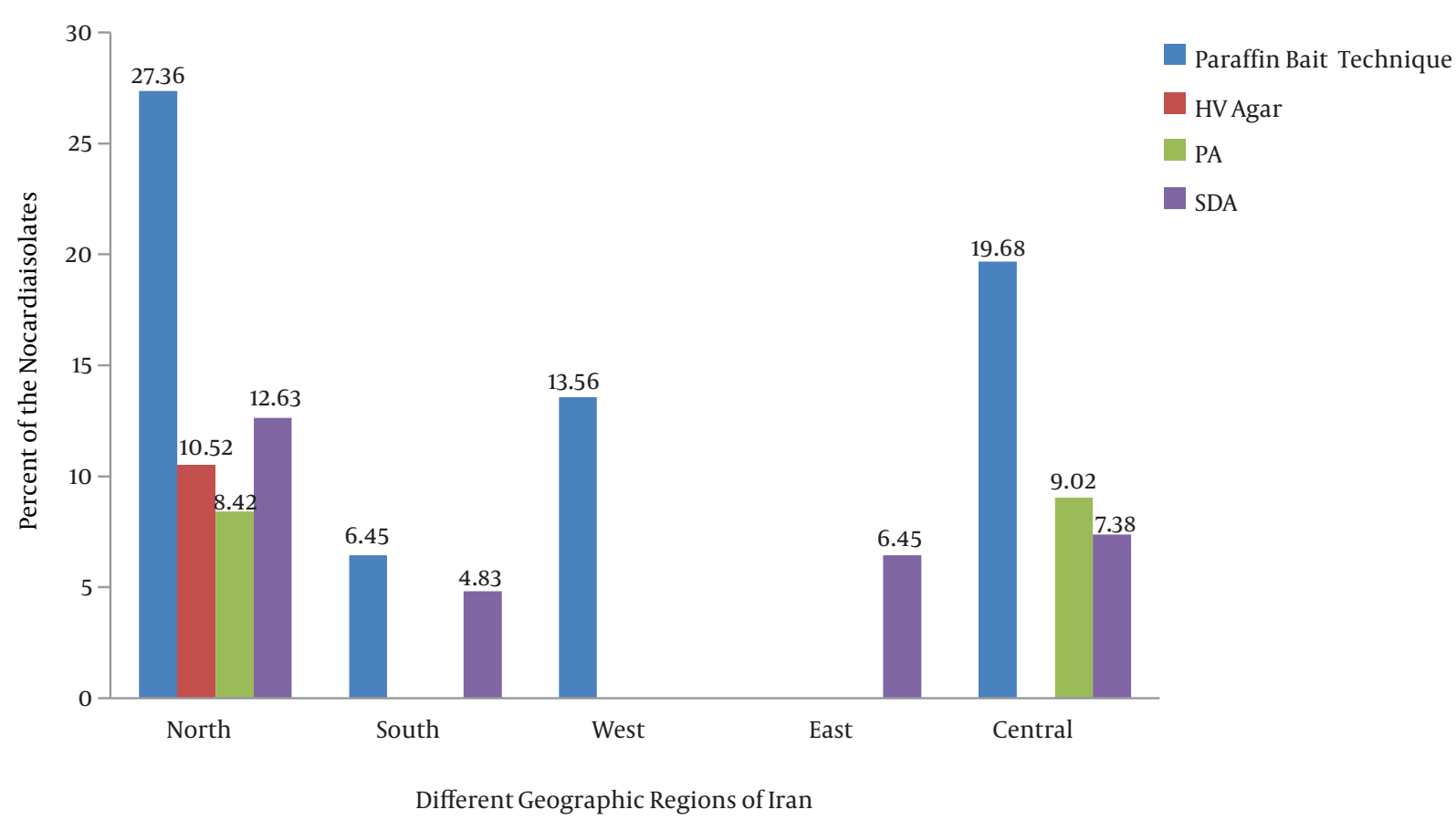

Figure 5. Percent of the Nocardia Isolates on Various Culture Media in Different Geographic Regions of Iran

recovery of Nocardia. Isolation of Nocardia species is enhanced by the use of paraffin baiting, which relies on the selective ability of the organism to metabolize paraffin.

\subsection{Conclusions}

Paraffin baiting technique due to less cost and more detection of colonies similar to Nocardia is better than others methods as a routine laboratory diagnostic procedure.

\section{Acknowledgments}

This study was supported by Tehran University of Medical Sciences, deputy of research.

\section{Footnotes}

'Contribution.2Authors'Contribution: None declared.

\section{Conflict of Interest: There is no Conflict of interest \\ Funding/Support: None declared.}

\section{References}

1. Brown-Elliott BA, Brown JM, Conville PS, Wallace RJ. Clinical and laboratory features of the Nocardia spp. based on current molecular taxonomy. Clin Microbiol Rev. 2006;19(2):259-82. doi: 10.1128/CMR.19.2.259-282.2006. [PubMed:16614249].
2. Valenzuela-Tovar JF, Contreras-Perez C, Shibayama-Hernandez $\mathrm{H}$, Chavez-Gonzalez L, Vazquez-Chacon CA, Olivera-Diaz H. Biochemical identification and molecular characterization (PCR-RFLP) of Nocardia isolates from sputum. Arch Med Res. 2005;36(4):356-61. doi: 10.1016/j.arcmed.2005.03.031. [PubMed: 15950074].

3. Conville PS, Fischer SH, Cartwright CP, Witebsky FG. Identification of nocardia species by restriction endonuclease analysis of an amplified portion of the 16S rRNA gene. J Clin Microbiol. 2000;38(1):158-64. [PubMed: 10618080].

4. Bafghi MF, Eshraghi SS, Heidarieh P, Habibnia S, Nasab MR. Nocardiosis in immune disorder disease. Malays J Med Sci. 2014;21(1):75-6. [PubMed: 24639618].

5. DeCroos FC, Garg P, Reddy AK, Sharma A, Krishnaiah S, Mungale M, et al. Optimizing diagnosis and management of nocardia keratitis, scleritis, and endophthalmitis: 11-year microbial and clinical overview. Ophthalmology. 2011;118(6):1193-200.doi:10.1016/j.ophtha.2010.10.037. [PubMed: 21276615].

6. Baumgardner DJ. Soil-related bacterial and fungal infections. J Am Board Fam Med. 2012;25(5):734-44. doi: 10.3122/jabfm.2012.05.110226. [PubMed: 22956709].

7. Hayakawa M, Nonomura H. Humic acid-vitamin agar, a new medium for the selective isolation of soil actinomycetes. J Ferment Technol. 1987;65(5):501-9. doi:10.1016/0385-6380(87)90108-7.

8. Mishra SK, Randhawa HS. Application of paraffin bait technique to the isolation of Nocardia asteroides from clinical specimens. Appl Microbiol. 1969;18(4):686-7. [PubMed: 4905040].

9. Yamamura $\mathrm{H}$, Hayakawa M, Iimura Y. Application of sucrose-gradient centrifugation for selective isolation of Nocardia spp. from soil. J Appl Microbiol. 2003;95(4):677-85. [PubMed: 12969279].

10. Eshraghi SS, Habibnia S, Heidarieh P, Rasouli Nasab M, Pourmand MR, Fatahi Bafghi M. Phenotypic characterization ofNocardiaspp. isolated from Iran soil microflora. Int J Environ Health Engin. 2015;4(1):20. doi: 10.4103/2277-9183.158388. 
11. Singh M, Sandhu RS, Randhawa HS. Comparison of paraffin baiting and conventional culture techniques for isolation of Nocardia asteroides from sputum. J Clin Microbiol. 1987;25(1):176-7. [PubMed: 3539990].

12. Wauters G, Avesani V, Charlier J, Janssens M, Vaneechoutte M Delmee M. Distribution of nocardia species in clinical samples and their routine rapid identification in the laboratory. J Clin Microbiol. 2005;43(6):2624-8. doi: 10.1128/JCM.43.6.2624-2628.2005. [PubMed: 15956375].

13. Goodfellow M. Characterisation of Mycobacterium, Nocardia, Corynebacterium and related taxa. Ann Soc Belg Med Trop. 1973;53(4):287-98. [PubMed: 4578844].

14. Goodfellow M, Lind A, Mordarska H, Pattyn S, Tsukamura M. A cooperative numerical analysis of cultures considered to belong to the 'rhodochrous' taxon. J Gen Microbiol. 1974;85(2):291-302. doi: 10.1099/00221287-85-2-291. [PubMed: 4217356].

15. Eshraghi SS. Molecular typing of Nocardia species. J Med Bacteriol. 2015;1(1-2):38-45.

16. Shawar RM, Moore DG, LaRocco MT. Cultivation of Nocardia spp. on chemically defined media for selective recovery of isolates from clinical specimens. J Clin Microbiol. 1990;28(3):508-12. [PubMed: 2182669].

17. Jaffe SM, Nash AG, Nasiri N. Nocardia asteroides: an unusual cause of breast abscess. Breast. 1999;8(6):345-6. doi: 10.1054/brst.1999.0073. [PubMed: 14731465].

18. Martin FJ, Perez-Bernal AM, Camacho F. Pemphigus vulgaris and disseminated nocardiosis.JEurAcad Dermatol Venereol. 2000;14(5):416-8. [PubMed: 11305389].

19. Igarashi M, Hayashi C, Homma Y, Hattori S, Kinoshita N, Hamada M, et al. Tubelactomicin A, a novel 16-membered lactone antibiotic, from Nocardia sp. I. Taxonomy, production, isolation and biological properties. J Antibiot (Tokyo). 2000;53(10):1096-101. [PubMed: 11132953].
20. Blackall LL, Barker SC, Hugenholtz P. Phylogenetic Analysis and Taxonomic History of Nocardia pinensis and Nocardia amarae. System Appl Microbiol. 1995;17(4):519-25. doi:10.1016/s0723-2020(11)80071-8.

21. Orchard VA, Goodfellow M. Numerical classification of some named strains of Nocardia asteroides and related isolates from soil. J Gen Microbiol. 1980;118(2):295-312. doi: 10.1099/00221287-118-2-295. [PubMed: 7003057].

22. Hayakawa M. Studies on the Isolation and Distribution of Rare Actinomycetes in Soil. Actinomycetologica. 2008;22(1):12-9. doi: 10.3209/saj.SAJ220103.

23. Garg P. Fungal, Mycobacterial, and Nocardia infections and the eye: an update. Eye (Lond). 2012;26(2):245-51. doi: 10.1038/eye.2011.332. [PubMed: 22173077].

24. Khan ZU, Neil L, Chandy R, Chugh TD, Al-Sayer H, Provost F, et al. Nocardia asteroides in the soil of Kuwait. Mycopathologia. 1997;137(3):15963. [PubMed: 9424591].

25. Ayyar S, Tendolkar U, Deodhar L. A comparison of three media for isolation of Nocardia species from clinical specimens. J Postgrad Med. 1992;38(2):70-2. [PubMed: 1432832].

26. Aghamirian MR, Ghiasian SA. Isolation and characterization of medically important aerobic actinomycetes in soil of iran (2006 - 2007). Open Microbiol J. 2009;3:53-7. doi: 10.2174/1874285800903010053. [PubMed: 19440253].

27. Fatahi Bafghi M, Eshraghi S, Abdollahi A, Negahban S, Ashrafi A, Heidarieh $\mathrm{P}$, et al. Isolation and Identification of Nocardia in Suspected Tuberculosis Samples Using Paraffin Baiting Technique. Sadra Med Sci J. 2014;2(2 Apr).

28. Gugnani HC, Unaogu IC, Emeruwa CN. Incidence of pulmonary infection due to Nocardia species in Nigeria. Mycoses. 1991;34(7-8):359-61. [PubMed: 1803243] 